\title{
On conclusive eavesdropping and measures of mutual information in quantum key distribution
}

\author{
Alexey E. Rastegin \\ Department of Theoretical Physics, Irkutsk State University, Gagarin Bv. 20, Irkutsk 664003, Russia
}

\begin{abstract}
We address the question of quantifying eavesdropper's information gain in an individual attack on systems of quantum key distribution. It is connected with the concept of conclusive eavesdropping introduced by Brandt. Using the BB84 protocol, we examine the problem of estimating a performance of conclusive entangling probe. The question of interest depends on the choice of a quantitative measure of eavesdropper's information about the error-free sifted bits. The FuchsPeres-Brandt probe realizes a very powerful individual attack on the BB84 scheme. In the usual formulation, Eve utilizes the Helstrom scheme in distinguishing between the two output probe states. In conclusive eavesdropping, the unambiguous discrimination is used. Comparing these two versions allows to demonstrate serious distinctions between widely used quantifiers of mutual information. In particular, the so-called Rényi mutual information does not seem to be a completely legitimate measure of an amount of mutual information. It is brightly emphasized with the example of conclusive eavesdropping.
\end{abstract}

PACS numbers: 03.67.Dd, 03.65.Ta

Keywords: BB84 protocol, quantum cryptographic entangling probe, Helstrom scheme, unambiguous state discrimination

\section{INTRODUCTION}

In last years of his scientific activity, Howard Brandt paid a significant attention to questions of quantum cryptography. Quantum cryptography provides a long-term solution to the problem of communication security [1-4] . In a series of papers [5-11], Brandt addressed the problem of optimizing eavesdropper's probe for attacking the BB84 protocol of quantum key distribution. The BB84 protocol [12] is probably the most known scheme of quantum key distribution. One of approaches to eavesdropping is connected with unambiguous state discrimination [7]. In a version of the B92 scheme, the two carriers are distinguished by the POVM for unambiguous state discrimination [1, 13]. A photonic implementation of such POVMs is considered in [14, 15]. The papers [7 9] addressed the case, when Eve tries to get data from her probe by means of unambiguous discrimination. It must be stressed that an efficiency of eavesdropping also depends on the used reconciliation method [16].

As was discussed in [7], the BB84 protocol may sometimes have a vulnerability analogous to the well-known vulnerability of the B92 protocol. The latter has further been considered in [17]. Here, the principal point concerns the effect of the inconclusive answer. To take it into account correctly, we should choose a proper informationtheoretic measure. Using the so-called "Rényi mutual information", the author of [17] found a certain weakness of the attack modification with unambiguous discrimination in comparison with the usual one. We will show that the amount of this weakness is essentially token in character. Indeed, it is considerably determined by the chosen entropic order in the measure of mutual information. The made comparison provides an interesting example, which helps to reveal some inadequacy of the widely used measure of mutual information. Since it reflects existing distinctions in information-theoretic properties, we also need in a detailed discussion of used quantities.

The contribution of this paper is two-fold. First, we aim to justify that the standard mutual information seems to be a preferred measure in estimating a performance of eavesdropper's probe. At least, it is better in studies of conclusive eavesdropping. Second, we motivate that the modified attack with unambiguous state discrimination is still of interest and deserves further investigations. Security of systems for quantum key distribution against eavesdropping is a difficult problem with many facets. Security analysis should take into account many scenarios possible in practice. To understand various aspects deeper, we prefer to consider them separately. In the present paper, we will focus on possible ways to quantify an information obtained by eavesdropper during an individual attack. Using inappropriate measure of information may lead to a wrong opinion about an essential weakness of conclusive eavesdropping.

The paper is organized as follows. In Section III, we discuss entropies and related information-theoretic terms. Considered properties are of great importance in interpretation of measures of mutual information. So, the property to show a reduction in the uncertainty of one random variable due to knowledge of other is based on the chain rule for the conditional entropy. In Section III] we briefly describe two versions of the Fuchs-Peres-Brandt (FPB) probe for eavesdropping the BB84 scheme. Main findings of this paper are presented in Section IV Using the two versions of the FPB probe, we compare their performance from the viewpoint of three measures of mutual information. In Section $\mathrm{V}$ we conclude the paper with a summary of results. 


\section{ENTROPIES AND RELATED NOTIONS OF INFORMATION THEORY}

In this section, we review some material from information theory. Quantum key distribution is a procedure used by Alice and Bob for obtaining two identical copies of a random and secret sequence of bits. Making some intrusion into a communication channel, Eve try to learn original bits. During this process, each of the three parties will obtain some string of bits. The three strings can be interpreted as binary random variables [13]. A degree of dependence between two random variables is typically measured in terms of the mutual information.

We begin with basic entropic functions. Let discrete random variable $X$ take values on finite set with the probability distribution $\{p(x)\}$. The Shannon entropy of $X$ is defined as [18]

$$
H(X):=-\sum_{x} p(x) \log p(x) .
$$

As a rule, the range of summation will be clear from the context. The logarithm in (1) is taken to the base 2 . Let $Y$ be another random variable, and let $p(x, y)$ denote corresponding joint probabilities. The joint entropy $H(X, Y)$ is defined like (1) by substituting the joint probabilities. An adequacy of the quantity (1) in applications to quantum measurement statistics was discussed by Brukner and Zeilinger [19]. Further development of their approach to information is considered in [20].

Dealing with the notion of conditional entropy, we introduce the function

$$
H(X \mid y):=-\sum_{x} p(x \mid y) \log p(x \mid y)
$$

where $p(x \mid y)=p(x, y) / p(y)$. Then the entropy of $X$ conditional on knowing $Y$ is defined as [18]

$$
H(X \mid Y):=\sum_{y} p(y) H(X \mid y) .
$$

The entropy is concave in probabilities, as the function $\xi \mapsto-\xi \log \xi$ is concave. It follows from concavity that [18]

$$
H(X \mid Y, Z) \leq H(X \mid Y) .
$$

In other words, conditioning on more reduces entropy. This property is essential for a treatment of (3) just as the conditional entropy. The definition (3) is further motivated by the chain rule [18]

$$
H(X, Y)=H(X \mid Y)+H(Y)=H(Y \mid X)+H(X) .
$$

It directly gives $H(X \mid Y)=H(X, Y)-H(Y)$, whence we see remaining lack of knowledge about $X$ at the given $Y$.

The notion of mutual information aims to measure how much information $X$ and $Y$ have in common [21]. The definition of mutual information is expressed as [18]

$$
I(X, Y):=H(X)+H(Y)-H(X, Y) .
$$

It is clearly symmetric in entries. By the chain rule (5), we can also rewrite (6) as

$$
I(X, Y)=H(X)-H(X \mid Y)=H(Y)-H(Y \mid X) .
$$

So, the mutual information shows a reduction in the uncertainty of one random variable due to knowledge of other [18]. In the context of quantum cryptography, the mutual information was first applied in [13]. Such an approach was motivated with using some ideas of the paper 22]. In the following, we will discuss some extensions of the above quantities to generalized entropic functions. Note that such generalizations typically lose some of the essential properties used in information theory. The mutual information (6) is also used in definition of the Shannon distinguishability [23, 24]. Fuchs gave a comprehensive presentation of distinguishability measures in information theory, with a list of 528 references on related topics [25].

Rényi entropies form an important family of one-parametric extensions of the Shannon entropy (1). For $0<\alpha \neq 1$, the Rényi entropy of order $\alpha$ is defined as [26]

$$
R_{\alpha}(X):=\frac{1}{1-\alpha} \log \left(\sum_{x} p(x)^{\alpha}\right)
$$

Rényi considered (8) in connection with formal postulates characterizing entropic functions [26]. The entropy (8) is a non-increasing function of $\alpha$ [26]. The entropy is maximized for the uniform distribution: if $p(x)=1 / N$ for all $x$ 
then (8) becomes $\log N$, or merely 1 for $N=2$. In the limit $\alpha \rightarrow 1$, the Rényi entropy gives (1). The joint $\alpha$-entropy $R_{\alpha}(X, Y)$ is defined in line with (8) by substituting the joint probabilities.

Some special choices of $\alpha$ are widely used in the literature. The limit $\alpha \rightarrow 0$ leads to the max-entropy equal to logarithm of the number of non-zero probabilities. In the binary case, non-trivial max-entropies are all equal to $\log 2=1$. Taking the limit $\alpha \rightarrow \infty$, we obtain the min-entropy $R_{\infty}(X)=-\log \max p(x)$. The case $\alpha=2$ gives the so-called collision entropy, which will play an important role in our discussion:

$$
R_{2}(X)=-\log \left(\sum_{x} p(x)^{2}\right)
$$

The question of concavity should be emphasized separately. For $\alpha \in[0 ; 1]$, the Rényi entropy is concave irrespectively to the actual dimensionality [27]. Convexity properties of $R_{\alpha}(X)$ with orders $\alpha>1$ depend on dimensionality of probabilistic vectors [28, 29]. For instance, the binary Rényi entropy is concave for $\alpha \in[0 ; 2][28]$.

To extend the notion of mutual information to the Rényi case, we should define the corresponding conditional form. There is no generally accepted definition of conditional Rényi's entropy [30]. Similarly to (2), for $0<\alpha \neq 1$ we introduce the function

$$
R_{\alpha}(X \mid y):=\frac{1}{1-\alpha} \log \left(\sum_{x} p(x \mid y)^{\alpha}\right) .
$$

For $\alpha=0$, we mean here logarithm of the number of non-zero conditional probabilities. The conditional $\alpha$-entropy is then defined by 31,33 ]

$$
R_{\alpha}(X \mid Y):=\sum_{y} p(y) R_{\alpha}(X \mid y)
$$

The limit $\alpha \rightarrow 1$ gives the standard conditional entropy (3). Further, the case $\alpha=\infty$ leads to the conditional min-entropy. For the given $y$, we define

$$
\hat{x}(y):=\operatorname{Arg} \max _{x} p(x \mid y)
$$

so that $p(x \mid y) \leq p(\hat{x} \mid y)$ for all possible $x$ at the fixed $y$. The formula (10) then reads

$$
R_{\infty}(X \mid y)=-\log p(\hat{x} \mid y)
$$

Substituting (13) into the right-hand side of (11), we get the conditional min-entropy $R_{\infty}(X \mid Y)$. Similarly to (8)), the quantity (10) is a non-increasing function of $\alpha$. Hence, we have $R_{\infty}(X \mid Y) \leq R_{\alpha}(X \mid Y)$ for all $\alpha \geq 0$.

We now ask for a behavior of (11) under conditioning on more. As a corollary of the concavity, for $\alpha \in[0 ; 1]$ we have 34

$$
R_{\alpha}(X \mid Y, Z) \leq R_{\alpha}(X \mid Y)
$$

The question is more difficult for $\alpha>1$. As mentioned in section 2.3 of [29], the Rényi entropy is not concave for $\alpha>\alpha_{*}>1$, where $\alpha_{*}$ depends on the number $N$ of possible outcomes. In the binary case, we can apply (14) for all positive orders up to $\alpha=2$. Unfortunately, no sufficiently exact estimations of the quantity $\alpha_{*}(N)$ are known. For more than two outcomes, we cannot use (14) for $\alpha=2$ and for larger values. Entropic functions of the Rényi type do not share some properties satisfied by the standard entropic functions. In particular, the conditional form (11) of order $\alpha \neq 1$ does not generally obey the chain rule. A comparison of proposed forms of conditional Rényi's entropy is given in [30].

We now proceed to a discussion of the so-called "Rényi mutual information". By an analogy with the formula (7), one introduces the quantity

$$
I_{\alpha}^{(R)}(X, Y):=R_{\alpha}(X)-R_{\alpha}(X \mid Y)
$$

It could be interpreted as Rényi's version of mutual information. With $\alpha=2$, this quantity is widely used in studying a performance of probes in individual attacks on quantum cryptographic systems [5, 7, 17, 35, 36]. Security against collective attacks is typically studied with using the Holevo information [37]. Despite of analogy between (7) and (15), the latter with $\alpha \neq 1$ is different in some essential respects. First, the quantity (15) is not generally symmetric in its entries. Second, the parameter $\alpha$ runs a continuum of values, so that a proper choice of its value is not a priori clear. In a certain sense, advantages of an approach with generalized entropic functions is rather associated with a possibility to vary the used parameter [32]. Third, the conditional $\alpha$-entropy (11) does not share the chain rule. 
Hence, we may fail with interpreting (15) as a reduction in the uncertainty of one random variable due to knowledge of other.

The above facts advise that we be very careful in treatment of the quantity (15), especially when only one value $\alpha \neq 1$ is involved. We will further use generally accepted term "Rényi's mutual information". Due to the above reasons, however, this quantity is not a completely legitimate measure of mutual information. For $\alpha>1$, we can rather interpret it as a family of upper bounds on the standard mutual information. In the considered model of key distribution, the bits are completely random and the scheme works symmetrically with respect to the values " 0 " and " 1 ". This results in the fact that an entropy of each binary variable per se reaches its maximum log $2=1$ irrespectively to $\alpha$. Let random variable $X$ with $N$ possible outcomes be distributed uniformly, i.e., $R_{\alpha}(X)=\log N$ independently of $\alpha$. For $\alpha>\beta$, we then have

$$
I_{\alpha}^{(R)}(X, Y) \geq I_{\beta}^{(R)}(X, Y)
$$

It follows from $R_{\alpha}(X \mid Y) \leq R_{\beta}(X \mid Y)$ for $\alpha>\beta$, as the Rényi entropy of $X$ is constant. Thus, Rényi's mutual information of order $\alpha>1$ gives an upper bound on the standard mutual information $I(X, Y)$. Properties of the above information measures will be essential in studies of their adequacy. The two versions of the FPB probe form a very spectacular example for considering this question.

\section{TWO VERSIONS OF THE FPB PROBE FOR EAVESDROPPING ON THE BB84 SCHEME}

In this section, we recall basic details of the BB84 protocol, which is most analyzed and most often implemented [3]. In this paper, we do not consider the problem of optimization of probe characteristics. Rather, we wish to compare several possible measures to estimate a performance of given probes. Two different approaches can be used on Eve's side during an attack on the BB84 protocol of quantum key distribution. In any case, the eavesdropper is asked by distinguishing a quantum state from several known alternatives. The two basic schemes of distinguishing non-identical pure states are referred to as the minimum error discrimination and the unambiguous discrimination.

The problem of optimization of entangling probe for an individual attack was considered by Fuchs and Peres [38]. They showed numerically that the optimal detection method for a two-level system can be obtained with a twodimensional probe only. The authors of [35] have made detailed analysis for the BB84 scheme [12]. In the paper [6], Brandt showed that the obtained probe for attacking the BB84 scheme can be realized with a single CNOT gate. Following [17, 36], this probe will be referred to as the Fuchs-Peres-Brandt (FPB) probe. The analysis of [35] is related to the case with the error-discard as reconciliation procedure. When Alice and Bob use other reconciliation methods, the probe considered is not optimal [16].

Let Alice and Bob use the two polarization bases $\{|h\rangle,|v\rangle\}$ and $\{|r\rangle,|\ell\rangle\}$. With respect to the horizontal polarization, the kets $|r\rangle$ and $|\ell\rangle$ of the diagonal basis relate to the angles $\pi / 4$ and $3 \pi / 4$, respectively. In each bit interval Alice sends a single photon prepared accordingly. Eve uses this photon as the control qubit input to a CNOT gate. Computational basis of this gate is defined in terms of the polarization states as

$$
\begin{aligned}
& |0\rangle=\cos (\pi / 8)|h\rangle+\sin (\pi / 8)|v\rangle, \\
& |1\rangle=-\sin (\pi / 8)|h\rangle+\cos (\pi / 8)|v\rangle .
\end{aligned}
$$

Eve prepares her own probe photon in the initial state

$$
\left|t_{i n}\right\rangle=c|+\rangle+s|-\rangle
$$

where $c=\sqrt{1-2 P_{E}}, s=\sqrt{2 P_{E}}$, and $| \pm\rangle=(|0\rangle \pm|1\rangle) / \sqrt{2}$. The introduced parameter $P_{E} \in[0 ; 1 / 2]$ turns out to be the error probability [17, 36]. The state (19) is input as the target qubit into Eve's CNOT gate controlled by sent Alice's qubit.

To find probabilities of outcomes, the output of the gate should be expressed as a superposition, in which the first qubit is represented in a proper basis. It will be convenient to introduce sub-normalized vectors

$$
\begin{aligned}
& \left|t_{ \pm}\right\rangle=c|+\rangle \pm \frac{s}{\sqrt{2}}|-\rangle, \\
& \left|t_{E}\right\rangle=\frac{s}{\sqrt{2}}|-\rangle .
\end{aligned}
$$

When Alice uses the basis $\{|h\rangle,|v\rangle\}$, Eve's CNOT gate acts as

$$
\begin{aligned}
&|h\rangle \otimes\left|t_{i n}\right\rangle \longmapsto|h\rangle \otimes\left|t_{+}\right\rangle+|v\rangle \otimes\left|t_{E}\right\rangle, \\
&|v\rangle \otimes\left|t_{i n}\right\rangle \longmapsto|v\rangle \otimes\left|t_{-}\right\rangle+|h\rangle \otimes\left|t_{E}\right\rangle .
\end{aligned}
$$


In a similar manner, for the basis $\{|r\rangle,|\ell\rangle\}$ we obtain

$$
\begin{aligned}
& |r\rangle \otimes\left|t_{i n}\right\rangle \longmapsto|r\rangle \otimes\left|t_{+}\right\rangle-|\ell\rangle \otimes\left|t_{E}\right\rangle, \\
& |\ell\rangle \otimes\left|t_{i n}\right\rangle \longmapsto|\ell\rangle \otimes\left|t_{-}\right\rangle-|r\rangle \otimes\left|t_{E}\right\rangle .
\end{aligned}
$$

Suppose that Bob applies the basis that Alice has employed and his outcome matches what Alice sent. This case focuses on the error-free sifted bits shared by Alice and Bob. To learn their shared bit value, Eve should distinguish between the sub-normalized outputs $\left|t_{+}\right\rangle$and $\left|t_{-}\right\rangle$of the target qubit. At this stage, she could apply two different approaches mentioned above.

In the Helstrom scheme of distinguishing between $\left|t_{+}\right\rangle$and $\left|t_{-}\right\rangle$, the average error probability is minimized [39, 40]. In the case considered, this measurement is described by projectors $|0\rangle\langle 0|$ and $|1\rangle\langle 1|$. For any of the states $\left|t_{+}\right\rangle$and $\left|t_{-}\right\rangle$, the false-alarm probability is equal to

$$
\frac{1}{2}\left(1-\frac{\sqrt{4 P_{E}\left(1-2 P_{E}\right)}}{1-P_{E}}\right) .
$$

The second approach is known as the unambiguous discrimination [41 43]. It sometimes gives an inconclusive answer, but never makes an error of mis-identification. Let us define two sub-normalized states

$$
\left|\tau_{ \pm}\right\rangle=\frac{s}{\sqrt{2}}|+\rangle \mp c|-\rangle
$$

so that $\left\langle\tau_{+} \mid t_{+}\right\rangle=\left\langle\tau_{-} \mid t_{-}\right\rangle=0$. The POVM elements of unambiguous discrimination are then written as [17]

$$
\mathrm{M}_{+}=\frac{1}{2 c^{2}}\left|\tau_{-}\right\rangle\left\langle\tau_{-}\left|, \quad \mathrm{M}_{-}=\frac{1}{2 c^{2}}\right| \tau_{+}\right\rangle\left\langle\tau_{+}\left|, \quad \mathrm{M}_{?}=\frac{2 c^{2}-s^{2}}{2 c^{2}}\right|+\right\rangle\langle+| .
$$

The probability of inconclusive answer is equal to the overlap of the states to be discriminated [43]. Calculating the overlap with the normalized vectors, we get

$$
\frac{c^{2}-s^{2} / 2}{c^{2}+s^{2} / 2}=\frac{1-3 P_{E}}{1-P_{E}}
$$

We shall now obtain values of any mutual information as a function of $P_{E}$.

To analyze a probe performance, calculations will be conditioned on the error-free sifted bits shared by Alice and Bob [17, 36]. Let us begin with the case, when Eve uses the Helstrom scheme. By the prime sign, we will mean terms related to the error-free sifted bits. It is also assumed that $0 \leq P_{E} \leq 1 / 3$. The formula (26) merely gives the conditional probabilities $p\left(e^{\prime}=0 \mid b^{\prime}=1\right)$ and $p\left(e^{\prime}=1 \mid b^{\prime}=0\right)$. As Alice's bits are equally likely to be 0 and 1 , two possible values of $b^{\prime}$ are equally likely as well. Multiplying (26) by $1 / 2$, we then obtain the joint probabilities $p\left(e^{\prime}, b^{\prime}\right)$ for $e^{\prime} \neq b^{\prime}$. The joint probabilities for the case $e^{\prime}=b^{\prime}$ read

$$
p\left(e^{\prime}=j, b^{\prime}=j\right)=\frac{1+\varkappa}{4}, \quad \varkappa=\frac{\sqrt{4 P_{E}\left(1-2 P_{E}\right)}}{1-P_{E}},
$$

where $j=0,1$. It is also clear that here we have the symmetry $p\left(e^{\prime} \mid b^{\prime}\right)=p\left(b^{\prime} \mid e^{\prime}\right)$. With these facts, we easily obtain any quantity representing an amount of mutual information. The standard mutual information is written as

$$
I\left(B^{\prime}, E^{\prime}\right)=\frac{1+\varkappa}{2} \log (1+\varkappa)+\frac{1-\varkappa}{2} \log (1-\varkappa) .
$$

The Rényi mutual information of order $\alpha=2$ becomes [17, 36$]$

$$
I_{2}^{(R)}\left(B^{\prime}, E^{\prime}\right)=\log \left(1+\varkappa^{2}\right)
$$

It is typically used for estimating a performance of quantum cryptographic probes [5, 7, 17, 35, 36]. At the same time, there are no fundamental reasons to prefer just the order $\alpha=2$. Advantages of a consideration with generalized entropies are rather connected with variations of entropic parameters. We will also include the case $\alpha=\infty$ due to (16). For the first version of the FPB probe, we have

$$
I_{\infty}^{(R)}\left(B^{\prime}, E^{\prime}\right)=\log (1+\varkappa) .
$$


This value shows the least point of the interval, in which the mutual $\alpha$-information ranges for the given $P_{E}$. Comparing the three quantities (31), (32), and (33) with the case of conclusive probe will show deficiencies of using (151) as a measure of Eve's information gain. The quantities (31), (32), and (33) are symmetric with respect to the entries $B^{\prime}$ and $E^{\prime}$. It is not the case for the probe with unambiguous discrimination.

In the conclusive modification, Eve discriminates between $\left|t_{+}\right\rangle$and $\left|t_{-}\right\rangle$unambiguously. This case is more complicated as involving an inconclusive answer. Restricted to the error-free sifted bits of Alice and Bob, the probabilities of outcomes are

$$
p\left(e^{\prime}=0\right)=p\left(e^{\prime}=1\right)=\frac{P_{E}}{1-P_{E}}, \quad p\left(e^{\prime}=?\right)=\frac{1-3 P_{E}}{1-P_{E}} .
$$

We further have $p\left(b^{\prime}=j \mid e^{\prime}=j\right)=1$ and $p\left(b^{\prime}=j \mid e^{\prime}=\right.$ ? $)=1 / 2$ for $j=0,1$ [17]. For the conclusive probe, the standard mutual information is equal to

$$
\widetilde{I}\left(B^{\prime}, E^{\prime}\right)=1-\frac{1-3 P_{E}}{1-P_{E}}=\frac{2 P_{E}}{1-P_{E}} .
$$

For all $\alpha \geq 0$, the Rényi mutual $\alpha$-information is also given by the right-hand side of (35). That is, it does not depend on $\alpha$. This property reflects that the situation is completely deterministic whenever $e^{\prime} \neq$ ? too. Hence, for all $\alpha \geq 0$ we have

$$
R_{\alpha}\left(B^{\prime} \mid e^{\prime}=0\right)=R_{\alpha}\left(B^{\prime} \mid e^{\prime}=1\right)=0 .
$$

For $e^{\prime}=$ ?, two possible values of $B^{\prime}$ are equiprobable, so that $R_{\alpha}\left(B^{\prime} \mid e^{\prime}=\right.$ ?) $=\log 2=1$. For all $\alpha \geq 0$, therefore, the conditional entropy reads

$$
R_{\alpha}\left(B^{\prime} \mid E^{\prime}\right)=p\left(e^{\prime}=?\right) R_{\alpha}\left(B^{\prime} \mid e^{\prime}=?\right)=\frac{1-3 P_{E}}{1-P_{E}} .
$$

Bob's bits are equally likely to be 0 and 1 , whence $R_{\alpha}\left(B^{\prime}\right)=\log 2=1$. Combining the latter with (37), we actually get

$$
\widetilde{I}_{\alpha}^{(R)}\left(B^{\prime}, E^{\prime}\right)=\frac{2 P_{E}}{1-P_{E}} .
$$

Thus, the Rényi mutual information increases with $\alpha$ for the usual probe and is constant for the conclusive probe. The above results will be used in analysis of an adequacy of Rényi's mutual information in the context of quantum cryptography.

\section{RÉNYI'S MUTUAL INFORMATION AS A MEASURE OF PROBE PERFORMANCE}

In this section, we consider quantities of the form (15) as estimators of a performance of quantum cryptographic probes. In particular, we will focus on the Rényi mutual information of order $\alpha=2$. The author of [17] examined the conclusive version of the FPB probe just on the ground of this measure. On the other hand, there is no fundamental reasons to prefer namely the order $\alpha=2$. Such choice of entropic parameter was rather used in [35] for simplifying analytical calculations. Indeed, explicit formulas of optimization problem seem to be most tractable for $\alpha=2$ too. However, some convenience of calculations hardly is a proper motivation to choose the basic figure of a probe performance. The standard mutual information (6) obey many nice properties that justify its usual interpretation. Such properties are connected with characteristics of the conditional entropy (3). The definition (111) of conditional Rényi's entropy is losing many of desired properties. In particular, it does not share the usual chain rule. Only for two outcomes we can be sure that conditioning reduces the entropy with orders up to 2 . For $\alpha>1$, the Rényi entropy is neither purely concave nor purely convex. The answer crucially depends on the number of possible outcomes [28]. In general, the Rényi mutual information is not symmetric in its entries. For the conclusive modification of the FPB probe, we generally have

$$
\widetilde{I}_{\alpha}^{(R)}\left(B^{\prime}, E^{\prime}\right) \neq \widetilde{I}_{\alpha}^{(R)}\left(E^{\prime}, B^{\prime}\right) \quad(\alpha \neq 1) .
$$

The left-hand side of (39) is always given by (38), whereas the right-hand side of (39) essentially depends on $\alpha$. Note that for the usual FPB probe we actually have the symmetry $I_{\alpha}^{(R)}\left(B^{\prime}, E^{\prime}\right)=I_{\alpha}^{(R)}\left(E^{\prime}, B^{\prime}\right)$. This result follows from the 


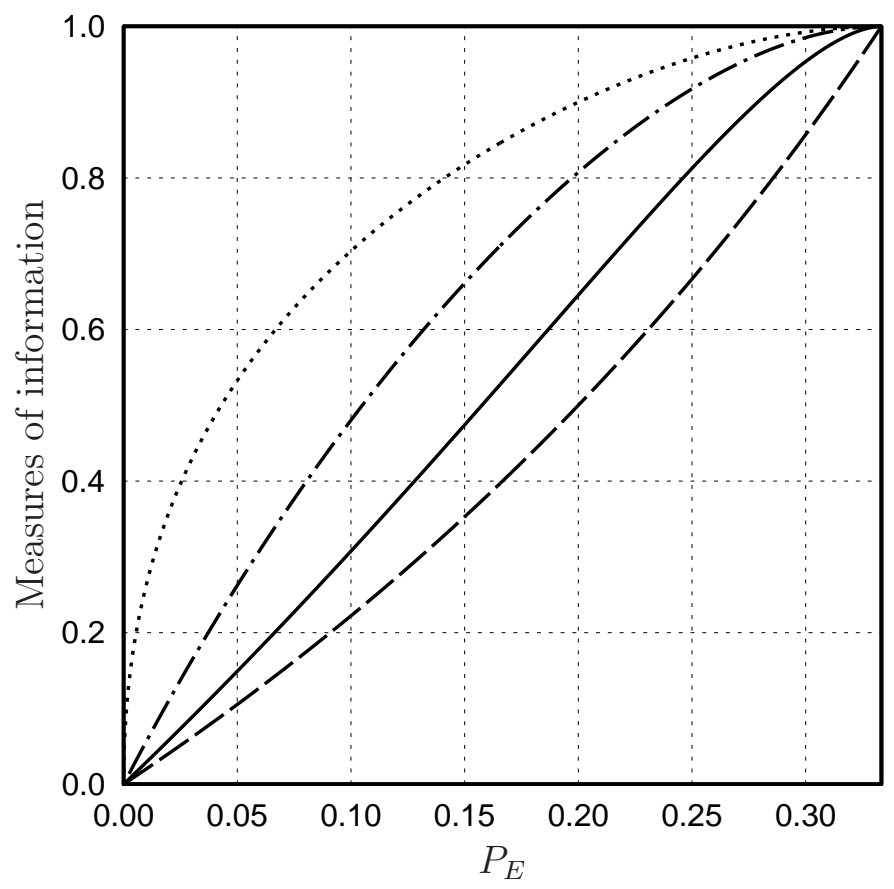

FIG. 1: For the FPB probe with projective measurement, it shows (31) by solid line, (32) by dash-dotted line, and (33) by dotted line. For the conclusive probe, it shows (38) by dashed line.

fact $p\left(b^{\prime} \mid e^{\prime}\right)=p\left(e^{\prime} \mid b^{\prime}\right)$ already mentioned for the probe with Helstrom's scheme of discrimination. In general, however, such symmetry is not valid.

The above reasons show difficulties with the quantity (15). At the same time, these reasons are rather general in character. To give a complete picture, we must mention one fact proved by the authors of [35]. For the original formulation of the B92 scheme, they proved that the standard mutual information and Rényi's information of order 2 are maximized by the same optimal probe. It may be not valid for sophisticated protocols or scenarios. Moreover, this property per se does not justify that Rényi's mutual information of order 2 is always able to replace the standard one. We shall now exemplify some inadequacy of (15) in the context of quantum cryptography. This conclusion is easily demonstrated by comparing the two versions of the FPB probe from the viewpoint of measures of the form (15) with several $\alpha$. Apparently, it is better represented by a contrast between the curves, which show various measures as functions of $P_{E}$. As usual, we will focus on the interval $0 \leq P_{E} \leq 1 / 3$.

On Fig. 1, the four curves are plotted. For the FPB probe with Helstrom's scheme, one shows (31) by solid line, (32) by dash-dotted line, and (33) by dotted line. For the conclusive FPB probe, we show all the measures of mutual information by dashed line. Including the quantity (33) with $\alpha=\infty$ allows to describe an interval, in which the Rényi mutual information may range with changing $\alpha$. In the paper [17], the two probes were compared on the base of Rényi's mutual information of order 2. As we see from Fig. 11 a difference between dash-dotted and dashed lines is sufficiently large and reaches the maximum about 0.314. On this ground, a performance of the conclusive probe seems to be weak too, even for small values of $P_{E}$. However, this difference can be changed by varying $\alpha$, since the quantity (38) is independent of $\alpha$ due to a specific character of the conclusive probe. If we choose $\alpha=\infty$, then a difference is even more essential and reaches the maximum about 0.482 .

However, our conclusions on security of cryptographic schemes should be independent of a sporadic choice. Keeping in mind the result (16), we conclude that Rényi's information of order $\alpha>1$ should rather be treated as an upper bound on the mutual information. It is clearly demonstrated by the solid, dotted and dash-dotted lines for the FPB probe. The standard measure (6) of mutual information obeys many properties including the relation (77). It is for this reason that it is to be treated as a reduction in the uncertainty of one random variable due to knowledge of other. On the other hand, there is no known analog of the result (7) with measures of the Rényi type. Moreover, we have arrived at the fact (39). To make a valid decision on Eve's mutual information, the standard mutual information is most appropriate. This conclusion is brightly illustrated by the curves of Fig. 1) Comparing the solid and dashed lines shows that the conclusive probe is certainly not so weak, as it could be imagined with the dash-dotted line. Despite of some non-optimality, the conclusive probe may nevertheless be of interest due to additional reasons.

It is instructive to look at the situation in slightly other perspective. As Bob's binary variable is uniformly 
distributed, we have $R_{\alpha}\left(B^{\prime}\right)=\log 2=1$ irrespectively to $\alpha$. Hence, the maximization of Eve's mutual information is equivalent to the minimization of the corresponding conditional entropy. In this regard, we see a similarity with one scenario of entropic uncertainty relations for successive measurement [44, 45]. We already mentioned that the definition (11) loses some properties that are natural for a measure of conditional entropy. Since the term $R_{\alpha}\left(B^{\prime} \mid E^{\prime}\right)$ does not increase with $\alpha$, for $\alpha>1$ it gives a lower bound on the conditional entropy $H\left(B^{\prime} \mid E^{\prime}\right)$. This point is a counterpart of the fact that Rényi's mutual information of order $\alpha>1$ leads to an upper bound on the standard one.

Using the measures (32) or (33), the legitimate users tend to be sure in an essential weakness of the conclusive version in comparison with the usual FPB probe. So, they could underestimate the degree of vulnerability with respect to conclusive eavesdropping. On the other hand, an overstated estimation of probe power can lead to a wrong situation with practical realization of a communication system. As was remarked in section VI.L of [2], the infinite security implies the infinite cost. Developing this observation, we may arrive at a conclusion. Characteristics of a real cryptographic system are actually determined by the taken compromise between several conflicting requirements such as an amount of provided security versus paid costs or technological tools. In this regard, we wish to estimate precisely a performance of possible probes.

As curves of Fig. 1 show, using Rényi's mutual information may create an illusory "power" in amount of Eve's mutual information. A degree of this illusion depends on the choice of $\alpha>1$. Such a wrong expression about too strong probe performance may lead to rejecting some feasible realizations. They could seemingly be evaluated as unexpectedly vulnerable, even though they are suitable in other respects. For small values of $P_{E}$, the ratio of (32) to (31) can approach 2 arbitrarily close. Hence, a quality of the usual FPB probe in comparison with the conclusive one may be illusory up to two times. The mentioned difference is most essential for "weak" eavesdropping, when Eve attempts to get a small amount of information while causing only a slight disturbance. Nevertheless, this example illustrates a conclusion that security requirement could be overstated spuriously on the base of inappropriate measures of mutual information.

In principle, we may try to adopt the right-hand side of (39), which is the $\alpha$-entropy of Eve's bits minus the $\alpha$-entropy conditioned on Bob's bits. Our example shows, however, that such measures of mutual information are completely inappropriate. For the conclusive version, we obtain the conditional entropies

$$
p\left(e^{\prime}=j \mid b^{\prime}=j\right)=\frac{2 P_{E}}{1-P_{E}}, \quad p\left(e^{\prime}=? \mid b^{\prime}=j\right)=\frac{1-3 P_{E}}{1-P_{E}} .
$$

where $j=0,1$. To illustrate an inadequacy of $\widetilde{I}_{\alpha}^{(R)}\left(E^{\prime}, B^{\prime}\right)$ for $\alpha \neq 1$, we calculate it for the least order $\alpha=\infty$. Using (34) and (40), we finally obtain

$$
\widetilde{I}_{\infty}^{(R)}\left(E^{\prime}, B^{\prime}\right)= \begin{cases}0 & \text { if } 0 \leq P_{E} \leq 1 / 5 \\ \log \left(2 P_{E}\right)-\log \left(1-3 P_{E}\right) & \text { if } 1 / 5<P_{E}<1 / 4 \\ 1 & \text { if } 1 / 4 \leq P_{E} \leq 1 / 3\end{cases}
$$

That is, this quantity sharply increases from 0 to 1 in a narrow interval of $P_{E}$. For $\alpha=2$, we also get the quantity

$$
\widetilde{I}_{2}^{(R)}\left(E^{\prime}, B^{\prime}\right)=\log \left(\frac{4 P_{E}^{2}+\left(1-3 P_{E}\right)^{2}}{2 P_{E}^{2}+\left(1-3 P_{E}\right)^{2}}\right) .
$$

The latter presents slightly smoothed variant of (41) and also has a narrow interval of sharp increasing. This behavior quite differs from (35). Thus, the measures (41) and (42) hardly represent anything with respect to the conclusive probe.

In the context of quantum cryptography, unambiguous state discrimination is also interesting in another respect. The rate of inconclusive tests is actually an additional check parameter. This fact was actually noticed in [13] with respect to the B92 scheme with conclusive receiver. For some of individual entangling-probe attacks against this setup, Eve's activity affects a rate of inconclusive outcomes. As was mentioned in [13], unchanged rate of inconclusive results cannot be treated as a security confirmation. On the other hand, unexpected variations of this rate are probably a witness of opposite activity. The same fact seems to be valid with respect to Eve's party. Observing surprise changes in inconclusive tests, Eve will rather decide that her opponents have made counter measures. Of course, any party may influence on a rate of inconclusive outcomes by means of the so-called fake states. However, such an approach will demand additional costs and more complicated equipment. From this viewpoint, the use of unambiguous discrimination is certainly of practical interest.

The following question is also related to conclusive eavesdropping. As was discussed in section 4 of [17], conclusive eavesdropping is especially interesting when there is loss between Alice's transmitter and Bob's receiver. Here, Eve can act as follows. She captures each carrier from Alice and recognizes it using the unambiguous state discrimination. Then she prepares qubit for transmission to Bob, whenever the test has given the conclusive answer. When the probability of 
conclusive result is higher than the transmissivity of the communication channel, the quantum cryptography seems to be inappropriately vulnerable. In the terminology of the paper [13], this strategy corresponds to opaque eavesdropping. It seems that opaque eavesdropping on the BB84 scheme has received less attention than it deserves. In any case, studies of such a kind should use properly chosen quantifier of mutual information.

\section{CONCLUSIONS}

Using the two versions of eavesdropping with the FPB probe, we compared different quantifiers of an amount of Eve's mutual information about the error-free sifted bits. The so-called Rényi mutual information, usually used with order 2, is not a completely legitimate measure of the mutual information. For $\alpha>1$, it should rather be interpreted as an upper bound on the standard mutual information. The standard mutual information must be considered as a preferable measure, at least in studies of conclusive eavesdropping. These conclusions were expected from a consideration of properties of related information-theoretic quantities. Further, we explicitly demonstrate our findings by comparison of the conclusive modification with the usual one. The use of inappropriate measures of mutual information could lead to wrong decisions in practical building a communication with quantum key distribution.

Finally, we shall briefly discuss possible alternatives to using information functions of the Rényi type. Tsallis' entropies [46] form another especially important family of generalized entropies. Two conditional forms of the Tsallis entropy were considered [47]. First of them obeys the chain rule of usual form, whence Tsallis' counterpart of the mutual information is arisen [47]. In principle, such measures of information could be applied in the context of eavesdropping. Another way is to use information metrics of this type. The standard conditional entropy leads to an information metric between random variables [48]. Each of two conditional forms of Tsallis' entropy also provides a legitimate metric [49], though the second one does not share the chain rule. In Section IV] we already noticed that maximizing mutual information reached with the FPB probe can be rewritten as minimizing the corresponding conditional entropy. So, it is of interest to study a probe performance from the viewpoint of information metrics.

[1] Lomonaco, S.J.: A quick glance at quantum cryptography, Cryptologia 23, 1-41 (1999)

[2] Gisin, N., Ribordy, G., Tittel, W., Zbinden, H.: Quantum cryptography. Rev. Mod. Phys. 74, 145-195 (2002)

[3] van Assche, G.: Quantum Cryptography and Secret-Key Distillation. Cambridge University Press, Cambridge (2006)

[4] Barnett, S.M.: Quantum Information. Oxford University Press, Oxford (2009)

[5] Brandt, H.E.: Optimum probe parameters for entangling probe in quantum key distribution. Quantum Inf. Process. 2, 37-79 (2003)

[6] Brandt, H.E.: Quantum-cryptographic entangling probe. Phys. Rev. A 71, 042312 (2005)

[7] Brandt, H.E.: Unambiguous state discrimination in quantum key distribution. Quantum Inf. Process. 4, 387-398 (2005)

[8] Brandt, H.E.: Conclusive eavesdropping in quantum key distribution. J. Opt. B: Quantum Semiclass. Opt. 7, S553-S556 (2005)

[9] Brandt, H.E., Myers, J.M.: Expanded conclusive eavesdropping in quantum key distribution. E-print arXiv:quant-ph/0509211 (2005)

[10] Brandt, H.E.: Alternative design for quantum cryptographic entangling probe. J. Mod. Opt. 53, 1041-1045 (2006)

[11] Brandt, H.E., Myers, J.M.: Expanded quantum cryptographic entangling probe. J. Mod. Opt. 53, 1927-1930 (2006)

[12] Bennett, C.H., Brassard, G.: Quantum cryptography: public key distribution and coin tossing. In: Proceedings of the IEEE International Conference on Computers, Systems, and Signal Processing, Bangalore, India, 175-179. IEEE, New York (1984)

[13] Ekert, A.K., Huttner, B., Palma, G.M., Peres A.: Eavesdropping on quantum cryptographical systems. Phys. Rev. A 50, 1047-1056 (1994)

[14] Huttner, B., Muller, A., Gautier, J.D., Zbinden, H., Gisin, N.: Unambiguous quantum measurement of nonorthogonal states. Phys. Rev. A 54, 3783-3789 (1996)

[15] Brandt, H.E.: Positive operator valued measure in quantum information processing. Am. J. Phys. 67, 434-439 (1999)

[16] Herbauts, I.M., Bettelli, S., Hübel, H., Peev, M.: On the optimality of individual entangling-probe attacks against BB84 quantum key distribution. Eur. Phys. J. D 46, 395-406 (2008)

[17] Shapiro, J.H.: Performance analysis for Brandt's conclusive entangling probe. Quantum Inf. Process. 5, 11-24 (2006)

[18] Cover, T.M., Thomas, J.A.: Elements of Information Theory. John Wiley \& Sons, New York (1991)

[19] Brukner, C̆., Zeilinger, A.: Conceptual inadequacy of the Shannon information in quantum measurements. Phys. Rev. A 63, $022113(2001)$

[20] Rastegin, A.E.: On the Brukner-Zeilinger approach to information in quantum measurements. Proc. R. Soc. A 471, $20150435(2015)$

[21] Nielsen, M.A., Chuang, I.L.: Quantum Computation and Quantum Information. Cambridge University Press, Cambridge (2000) 
[22] Csiszár, I., Körner, J.: Broadcast channels with confidential messages. IEEE Trans. Inf. Theory 24, 339-348 (1978)

[23] Fuchs, C.A., van de Graaf, J.: Cryptographic distinguishability measures for quantum mechanical states. IEEE Trans. Inf. Theory 45, 1216-1227 (1999)

[24] Biham, E., Boyer, M., Brassard, G., van de Graaf, J., Mor, T.: Security of quantum key distribution against all collective attacks. Algorithmica 34, 372-388 (2002)

[25] Fuchs, C.A.: Distinguishability and accessible information in quantum theory. E-print arXiv:quant-ph/9601020 (1996)

[26] Rényi, A.: On measures of entropy and information. In: Neyman, J. (ed.) Proceedings of 4th Berkeley Symposium on Mathematical Statistics and Probability. Vol. I, 547-561. University of California Press, Berkeley (1961)

[27] Jizba, P., Arimitsu, T.: The world according to Rényi: thermodynamics of multifractal systems. Ann. Phys. 312, 17-59 (2004)

[28] Ben-Bassat, M., Raviv, J.: Rényi's entropy and error probability. IEEE Trans. Inf. Theory 24, 324-331 (1978)

[29] Bengtsson, I., Życzkowski, K.: Geometry of Quantum States: An Introduction to Quantum Entanglement. Cambridge University Press, Cambridge (2006)

[30] Teixeira, A., Matos, A., Antunes, L.: Conditional Rényi entropies. IEEE Trans. Inf. Theory 58, 4273-4277 (2012)

[31] Cachin, C.: Entropy measures and unconditional security in cryptography. Ph.D. thesis, Swiss Federal Institute of Technology, Zürich (1997)

[32] Kamimura, R.: Minimizing $\alpha$-information for generalization and interpretation. Algorithmica 22, 173-197 (1998)

[33] Erdogmus, D., Principe, J.C.: Lower and upper bounds for misclassification probability based on Rényi's information. J. VLSI Signal Process. 37, 305-317 (2004)

[34] Rastegin, A.E.: Further results on generalized conditional entropies. RAIRO-Theor. Inf. Appl. 49, 67-92 (2015)

[35] Slutsky, B.A., Rao, R., Sun, P.-C., Fainman, Y.: Security of quantum cryptography against individual attacks. Phys. Rev. A 57, 2383-2398 (1998)

[36] Shapiro, J.H., Wong, F.N.C.: Attacking quantum key distribution with single-photon two-qubit quantum logic. Phys. Rev. A 73, $012315(2006)$

[37] Scarani, V., Bechmann-Pasquinucci, H., Cerf, N.J., Dušek, M., Lütkenhaus, N., Peev, M.: The security of practical quantum key distribution. Rev. Mod. Phys. 81, 1301-1350 (2009)

[38] Fuchs, C.A., Peres, A.: Quantum state disturbance versus information gain: uncertainty relations for quantum information. Phys. Rev. A 53, 2038-2045 (1996)

[39] Helstrom, C.W.: Detection theory and quantum mechanics. Inform. Control 10, 254-291 (1967)

[40] Helstrom, C.W.: Quantum Detection and Estimation Theory. Academic Press, New York (1976)

[41] Ivanovic, I.D.: How to differentiate between non-orthogonal states. Phys. Lett. A 123, 257-259 (1987)

[42] Dieks, D.: Overlap and distinguishability of quantum states. Phys. Lett. A 126, 303-306 (1988)

[43] Peres, A.: How to differentiate between non-orthogonal states. Phys. Lett. A 128, 19 (1988)

[44] Baek, K., Farrow, T., Son, W.: Optimized entropic uncertainty for successive projective measurements. Phys. Rev. A 89, 032108 (2014)

[45] Zhang, J., Zhang, Y., Yu, C.-S.: Rényi entropy uncertainty relation for successive projective measurements. Quantum Inf. Process. 14, 2239-2253 (2015)

[46] Tsallis, C.: Possible generalization of Boltzmann-Gibbs statistics. J. Stat. Phys. 52, 479-487 (1988)

[47] Furuichi, S.: Information-theoretical properties of Tsallis entropies. J. Math. Phys. 47, 023302 (2006)

[48] Bennett, C.H., Gács, P., Li, M., Vitányi, P.M.D., Zurek, W.H.: Information distance. IEEE Trans. Inf. Theory 44, 1407-1423 (1998)

[49] Rastegin, A.E.: On generalized entropies and information-theoretic Bell inequalities under decoherence. Ann. Phys. 355, $241-257(2015)$ 